\title{
PEDIATRIC MR UROGRAPHY: INDICATIONS, TECHNIQUES AND ROLE IN URORADIOLOGY
}

\author{
Dubravko BOBINEC, Goran ROIĆ \\ Department of Pediatric Radiology \\ Children's Hospital Zagreb, Croatia
}

\author{
Correspondence: \\ dubravkob90@hotmail.com \\ Tel.: + 38514600234 \\ Fax.: + 38514600113
}

Received: May 13, 2016

Accepted: September 3, 2016

Key words: $\mathrm{fMRU}$ - Analysis • Urinary system - Function.

\begin{abstract}
The aim of writing the article is to bring closer to readers the role of this specific procedure in uroradiology. Functional magnetic resonance urography (fMRU) gives us an insight into the morphology of the urinary system with functional parameters similar to nuclear medicine (renal scintigraphy). MR urography (MRU) and functional (fMRU) provide both morphological and functional information by means of different sequences with or without injection of gadolinium. Combined static and dynamic contrast-enhanced MR urography is particularly advantageous in the pediatric population, offering high spatial resolution, morphological imaging of the urinary tract with reliable information about kidney function and urinary excretion in a single examination, without exposure to ionizing radiation. An fMRU scan requires external postprocessing using special dedicated software. The functional analysis is based primarily on the post-contrast sequences. Dynamic scans are repeated over 15 minutes with increasing pauses between acquisitions. The analytical results include: calyceal transit time, renal transit time, time-to-peak, whole (renal) volume, parenchymal (renal) volume, differential renal function, enhancement curves, excretion curves, and patlak plots. Conclusion - This is a noninvasive procedure without the use of ionizing radiation, providing a very detailed urinary tract anatomy and functional analysis of kidney function and urinary excretion.
\end{abstract}

Functional magnetic resonance urography (fMRU) gives us insight into the morphology of the urinary system with functional results, similar to nuclear medicine (renal scintigra$p h y)$. Even small kidney dysfunction can be detected by functional analysis software. In ureteropelvic obstruction, fMRU was comparable to the other imaging techniques, but it was superior modality concerning the evaluation of end-ureteral anomalies (1). With numerous advantages and possibilities for further development it is important to spread the knowledge about this procedure.

The functional analysis of the MRU scans requires external postprocessing using special dedicated software. One of such freewares is called „CHOP-fMRU“, developed by the The Children's Hospital of Philadelphia (2). A prerequisite for an optimal comprehensive analysis is an optimal scan which assee in Figure 1 (3).

The preparation of patients for MRU does not require any bowel cleansing or intake of laxative. On the day of examination patient is fasting. The intravenous hydration before the scanning is crucial for reducing the concentration of the contrast agent in the renal pelvis and for optimal distention of the urinary tract. The administration of furosemide 15 minutes prior to the contrast agent 


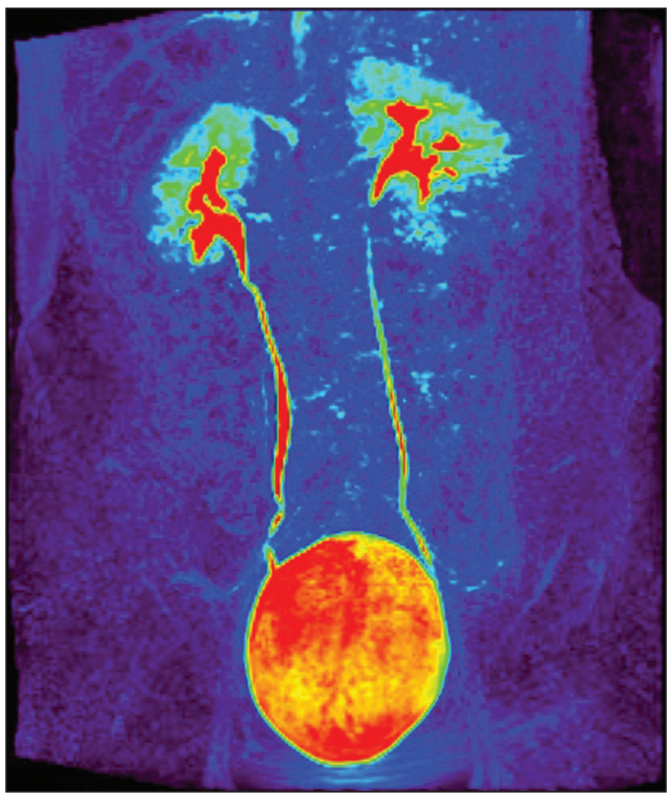

Figure 1 Color coded contrast MRI picture of urinary system from CHOP-fMRU software.

injection has the same purpose in addition to servin as the stress test for the the ureteropelvic junction $(4,5)$. Optimally the patient is scanned in the prone position (6). The functional analysis is based primarily on the post-contrast dynamic sequences (the regular dose of contrast agent is $0.1 \mathrm{mmol} / \mathrm{kg}$ gadolinium-DTPA) (4). For the post-contrast scan we employ 3-D T1-weighted gradient echo sequence with fat saturation ("volumetric interpolated breath-hold examination" - VIBE - Siemens ${ }^{\oplus}$, Erlangen, Germany) with the adjustment of parameters for a single series not to exceed 11 seconds and with each series have the same number of slices. The dynamic scan is repeated over 8 minutes with increasing pauses between acquisitions. When the excretion of contrast into the ureter is delayed additional single series of the urinary tract can be acquired at longer intervals. After the scan is completed, images are sent to external workstation for post-processing. This includes morphologic postprocessing evaluation of both the pre- and post-contrast 3-D scans in maximum-intensity projections
(MIP) and reformatting 3D volumetric representations (2). We analyze and use the following functional parameters:

- Calyceal transit time (CTT): the time needed for the contrast agent to reach the calyces from the time it appears in the aorta 4).

- Renal transit time (RTT): the time needed for the contrast agent to reach the ureter below the level of the lower pole of the kidney from the time it appears in the aorta (4).

- Time-to-peak (TTP): The time to reach maximal parenchymal enhancement,

- Whole (renal) volume: 3-D volume of the renal parenchyma and pelvicalyceal system.

- (Renal) parenchymal volume: 3-D volume of the contrast-enhanced renal parenchyma excluding the calyces and renal pelvis.

- Differential renal function (DRF): same as split renal function on renal scintigraphy and expressed for each kidney as percentage of the total renal function.

This can be based on different parameters:

a. Volumetric differential renal function (vDRF): split renal function based on the enhancing renal parenchymal volumes converted into percentages out of $100 \%$.

b. Patlak differential renal function (pDRF): split renal function based on the generated Patlak numbers, a potential indicator of the glomerular filtration rate (GFR).

c. Volumetric and Patlak differential renal function (vpDRF): Split renal function based on both the enhancing renal parenchymal volumes and Patlak numbers. This has proved to be useful in the case of a small kidney with high Patlak number to assess its overall relative functional contribution (Table 1) (2).

Enhancement curves - display the change in signal intensity over time (minutes) in the 


\section{Table 1 fMRU analytical results from CHOP-fMRU software (2)}

\begin{tabular}{lll}
\hline Renal values & Right Kidney & Left Kidney \\
\hline CTT & $0 \min (\mathrm{s}) 55 \mathrm{sec}(\mathrm{s})$ & $0 \mathrm{~min}(\mathrm{~s}) 13 \mathrm{sec}(\mathrm{s})$ \\
RTT & $2 \min (\mathrm{s}) 46 \mathrm{sec}(\mathrm{s})$ & $2 \mathrm{~min}(\mathrm{~s}) 41 \mathrm{sec}(\mathrm{s})$ \\
TTP & $2 \min (\mathrm{s}) 46 \mathrm{sec}(\mathrm{s})$ & $2 \mathrm{~min}(\mathrm{~s}) 46 \mathrm{sec}(\mathrm{s})$ \\
Whole volume $(\mathrm{ml})$ & $156.2 \mathrm{ml}$ & $279.3 \mathrm{ml}$ \\
Parenchymal volume (ml) & $73.87 \mathrm{ml}$ & $68.15 \mathrm{ml}$ \\
$\quad$ 6a. vDRF & $52.01 \%$ & $47.98 \%$ \\
6b. pDRF & $50.98 \%$ & $49.01 \%$ \\
$\quad$ 6c. vpDRF & $52.99 \%$ & $47.00 \%$ \\
Difference vDRF pDRF & $1.028 \%$ & $1.028 \%$ \\
Patlak $(\mathrm{ml} / \mathrm{min}) / \mathrm{ml}$ & 0.54013 & 0.51928 \\
BSA Patlak $(\mathrm{ml} / \mathrm{min}) / \mathrm{ml}$ & 0.47838 & 0.45991 \\
\hline
\end{tabular}

$\mathrm{CTT}=$ Calyceal transit time; RTT=Renal transit time; TTP=Time to peak; vDRF=Volumetric differential renal function; $\mathrm{pDRF}=$ Patlak differential renal fuction; $\mathrm{vpDRF}=$ Volumetric Patlak differential renal function.

segmented part of the aorta $(r e d)$ and the right (blue) and left (green) renal parenchymas. Excretion curves - the excretion curves display the change in relative signal intensity over time (minutes) in the enhanced segmented part of the pelvicalyceal system. Patlak plots - this is based on the Patlak-Rutland model, which is a graphical analysis technique based on the compartment model that uses linear regression to identify and analyse pharmacokinetics of tracers involving irreversible uptake (2).

\section{Conclusion}

fMRU is noninvasive procedure without the utilization of ionizing radiation that providies very detailed diagnostic anatomy and functional analysis of urinary system. This method overcomes a lot of limitations of conventional imaging modalities and has a potential to become a leading modality in paediatric uroradiology.

Authors' contributions: Conception and design: DB and GR; Acquisition, analysis and interpretation of data: DB and GR; Drafting the article: DB; Revising the article critically for intellectual content: DB and
GR; Approved final version of the manuscript: DB, and GR.

Conflict of interest: The authors declare that they have no conflict of interest.

\section{References}

1. Hadjidekov G, Hadjidekova S, Tonchev Z, Bakalova $R$, Aoki I. Assessing renal function in children with hydronephrosis - additional feature of MR urography. Radiol Oncol. 2011;45(4):248-9.

2. Khrichenko D, Darge K. Functional analysis in MR urography - made simple. Pediatr Radiol. 2010;40:182-99.

3. Leyendecker JR, Barnes CE, and Zagoria RJ. MR urography: techniques and clinical applications. Radiographics. 2008;28(1):23-46.

4. Grattan-Smith JD, Little SB, and Jones RA. MR urography in children: how we do it. Pediatr Radiol. 2008;38(1):3-17.

5. Grattan-Smith, JD, Perez-Bayfield MR, Jones RA, Little S, Broecker B, Smith EA, et al. MR imaging of kidneys: functional evaluation using F-15 perfusion imaging. Pediatr Radiol. 2003;33(5):293304.

6. Delgado J, Bedoya MA, Adeb M, Carson RH, Johnson AM, Khrichenko D, et al. Pediatr Radiol. 2015;45(9):1333-43. 